\title{
Positive Solutions for a Fourth-Order Boundary Value Problem
}

\author{
Kun Wang and Zhilin Yang \\ Department of Mathematics, Qingdao Technological University, Fushun Road No. 11, Quingdao, Shandong 266033, China
}

Correspondence should be addressed to Kun Wang; wangkun880304@163.com

Received 24 August 2012; Accepted 29 October 2012

Academic Editor: Andrea Pascucci

Copyright (c) $2013 \mathrm{~K}$. Wang and Z. Yang. This is an open access article distributed under the Creative Commons Attribution License, which permits unrestricted use, distribution, and reproduction in any medium, provided the original work is properly cited.

This paper deals with the existence and multiplicity of positive solutions for the fourth-order boundary value problem $u^{(4)}=$ $f\left(t, u, u^{\prime},-u^{\prime \prime}, u^{\prime \prime \prime}\right), u(0)=u^{\prime}(1)=u^{\prime \prime \prime}(0)=u^{\prime \prime}(1)=0$. Here $f \in C\left([0,1] \times \mathbb{R}_{+}^{4}, \mathbb{R}_{+}\right)\left(\mathbb{R}_{+}:=[0,+\infty)\right)$. We use fixed point index theory to establish our main results based on a priori estimates achieved by utilizing some integral identities and integral inequalities.

\section{Introduction}

The purpose of this paper is to study the existence and multiplicity of positive solutions for the fourth-order boundary value problem

$$
\begin{gathered}
u^{(4)}=f\left(t, u, u^{\prime},-u^{\prime \prime}, u^{\prime \prime \prime}\right), \\
u(0)=u^{\prime}(1)=u^{\prime \prime \prime}(0)=u^{\prime \prime}(1)=0,
\end{gathered}
$$

where $f \in C\left([0,1] \times \mathbb{R}_{+}^{4}, \mathbb{R}_{+}\right)$. By a positive solution of (1), we mean a function $u \in C^{4}\left([0,1], \mathbb{R}_{+}\right)$that solves (1) and satisfies $u(t)>0$ for all $t \in(0,1]$.

In recent years, fourth-order boundary value problems for nonlinear ordinary differential equations have been extensively studied by using diverse methods, including fixed point theorems on cones [1-6], the method of lower and upper solutions [7-10], the iterative method [11-14], critical point theory [15], and the shooting method [16]. It should be remarked that none of the results cited above involves derivatives of all orders in their nonlinearities. In [9], $\mathrm{Ma}$ and Yang studied the fourth-order four-point boundary value problem

$$
\begin{gathered}
u^{(4)}=f\left(t, u,-u^{\prime \prime}\right), \\
u(0)=0, \quad u(1)=a u(\eta), \\
u^{\prime \prime}(0)=0, \quad u^{\prime \prime}(1)=b u(\xi),
\end{gathered}
$$

where $\eta, \xi \in(0,1), a, b \geqslant 0$. The authors used the method of upper and lower solutions and a new maximum principle to establish their existence results. In [14], Pei and Chang studied a class of fourth-order boundary value problem

$$
\begin{gathered}
y^{(4)}=f(t, y), \\
y(0)=y(1)=y^{\prime}(0)=y^{\prime}(1)=0 .
\end{gathered}
$$

By using the monotone iterative technique, the authors proved that problem (3) has at least one symmetric positive solution under certain conditions. In [17], Pang et al. studied the existence and multiplicity of nontrivial solutions for the fourth-order boundary value problem

$$
\begin{gathered}
x^{(4)}=f\left(x,-x^{\prime \prime}\right), \\
x(0)=x(1)=x^{\prime \prime}(0)=x^{\prime \prime}(1)=0,
\end{gathered}
$$

where $f \in C\left(\mathbb{R}^{2}, \mathbb{R}\right)$. Making use of the theory of LeraySchauder degree, under appropriate conditions on the nonlinearity $f$, the authors proved that problem (4) has at least six different nontrivial solutions, including two positive ones and two negative ones.

In [18], Yang and Sun studied the fourth-order boundary value problem

$$
\begin{gathered}
u^{(4)}=f\left(t, u, u^{\prime},-u^{\prime \prime},-u^{\prime \prime \prime}\right), \\
u(0)=u^{\prime}(1)=u^{\prime \prime}(0)=u^{\prime \prime \prime}(1)=0,
\end{gathered}
$$


where $f \in C\left([0,1] \times \mathbb{R}_{+}^{4}, \mathbb{R}_{+}\right)$. By using fixed point index theory based on a priori estimates, the authors established the main results on the existence, multiplicity and uniqueness of positive solutions for (5).

Motivated by [18], in this paper, we will discuss the existence and multiplicity of positive solutions for problem (1). As in [18], we first use the method of order reduction to transform (1) into a boundary value problem for a secondorder integro-differential equation and then seek the existence and multiplicity of positive solutions for the resultant problem. To overcome the difficulties stemming from the presence of derivatives of all orders and the difference between (1) and (5) in their boundary value conditions, we have to prove that the maximum of every nonnegative concave function $v$ can be dominated by the integrals

$$
\begin{aligned}
& \int_{0}^{1} v(t)(1-t) e^{1-t} \mathrm{~d} t \\
& \int_{0}^{1}\left(\left(B_{1} v\right)(t)+2\left(B_{1} v\right)^{\prime}(t)\right)(1-t) e^{1-t} \mathrm{~d} t
\end{aligned}
$$

(see Lemmas 5 and 6 below for more details). Based on a priori estimates achieved by utilizing some integral identities and integral inequalities, we use fixed point index theory to prove the existence and multiplicity of positive solutions for (1).

This paper is organized as follows. In Section 2, we transform (1) into a boundary value problem for a secondorder integro-differential equation and then establish some basic integral identities and integral inequalities that are useful in deriving the priori estimates in the next section. Our main results, namely Theorems $10-12$, are stated and proved in Section 3.

\section{Preliminaries}

Let $E:=C^{1}([0,1], \mathbb{R})$ and $\|u\|:=\max \left\{\|u\|_{0},\left\|u^{\prime}\right\|_{0}\right\}$, where $\|u\|_{0}:=\max \{|u(t)|: t \in[0,1]\}$. Furthermore, let

$$
P:=\left\{u \in E: u(t) \geqslant 0, u^{\prime}(t) 0, \forall t \in[0,1]\right\} .
$$

Clearly $(E,\|\cdot\|)$ is a real Banach space and $P$ is a cone in $E$.

Let

$$
\begin{aligned}
& k_{1}(t, s):=\min \{t, s\}, \\
& k_{2}(t, s):=\min \{1-s, 1-t\} .
\end{aligned}
$$

Define the linear integral operators $B_{1}$ and $B_{2}$ by

$$
\left(B_{i} v\right)(t):=\int_{0}^{1} k_{i}(t, s) v(s) \mathrm{d} s, \quad v \in P(i=1,2) .
$$

Substituting $v(t):=-u^{\prime \prime}(t)$ into (1), we transform (1) into the following second-order boundary value problem for the integro-differential equation

$$
\begin{gathered}
-v^{\prime \prime}(t)=f\left(t,\left(B_{1} v\right)(t),\left(B_{1} v\right)^{\prime}(t), v(t),-v^{\prime}(t)\right), \\
v^{\prime}(0)=v(1)=0,
\end{gathered}
$$

which is equivalent to the nonlinear integral equation

$v(t)$

$$
=\int_{0}^{1} k_{2}(t, s) f\left(s,\left(B_{1} v\right)(s),\left(B_{1} v\right)^{\prime}(s), v(s),-v^{\prime}(s)\right) \mathrm{d} s .
$$

Define the operator $A: P \rightarrow P$ by

$(A v)(t)$

$$
\begin{array}{r}
:=\int_{0}^{1} k_{2}(t, s) f\left(s,\left(B_{1} v\right)(s),\left(B_{1} v\right)^{\prime}(s), v(s),-v^{\prime}(s)\right) \mathrm{d} s, \\
v \in P .
\end{array}
$$

Now $f \in C\left([0,1] \times \mathbb{R}_{+}^{4}, \mathbb{R}_{+}\right)$implies that $A$ is a completely continuous operator. In our setting, the existence of positive solutions for (1) is equivalent to that of positive fixed points of $A: P \rightarrow P$.

To establish the priori estimates of positive solutions for some problems associated with (11), we need several integral identities and integral inequalities below.

Lemma 1. If $v \in P \cap C^{2}[0,1], v^{\prime}(0)=v(1)=0$, then

$$
\int_{0}^{1}-v^{\prime \prime}(t)(1-t) e^{1-t} \mathrm{~d} t=\int_{0}^{1}\left(v(t)-2 v^{\prime}(t)\right)(1-t) e^{1-t} \mathrm{~d} t .
$$

Proof. Integrating by parts and using $v^{\prime}(0)=v(1)=0$, we have

$$
\begin{aligned}
& \int_{0}^{1}\left(-v^{\prime \prime}(t)\right)(1-t) e^{1-t} \mathrm{~d} t \\
& =\int_{0}^{1} v^{\prime}(t)(t-2) e^{1-t} \mathrm{~d} t \\
& =\int_{0}^{1}\left(-v^{\prime}(t)\right) t e^{1-t} \mathrm{~d} t-2 \int_{0}^{1} v^{\prime}(t)(1-t) e^{1-t} \mathrm{~d} t \\
& \quad \int_{0}^{1}-v^{\prime}(t) t e^{1-t} \mathrm{~d} t=\int_{0}^{1} v(t)(1-t) e^{1-t} \mathrm{~d} t
\end{aligned}
$$

from which (13) follows. This completes the proof.

Lemma 2. If $v \in C\left([0,1], \mathbb{R}_{+}\right)$, then

$$
\int_{0}^{1}\left(\left(B_{1} v\right)(t)+2\left(B_{1} v\right)^{\prime}(t) t e^{t} \mathrm{~d} t\right)=\int_{0}^{1} v(t) t e^{t} \mathrm{~d} t .
$$

Proof. Notice $\left(B_{1} v\right)(0)=0$. Integrating by parts, we obtain

$$
\int_{0}^{1}\left(B_{1} v\right)(t) t e^{t} \mathrm{~d} t=\int_{0}^{1}(1-t) e^{t}\left(B_{1} v\right)^{\prime}(t) \mathrm{d} t,
$$

so that

$$
\int_{0}^{1}\left(\left(B_{1} v\right)(t)+2\left(B_{1} v\right)^{\prime}(t)\right) t e^{t} \mathrm{~d} t=\int_{0}^{1}(1+t) e^{t}\left(B_{1} v\right)^{\prime}(t) \mathrm{d} t .
$$


Notice $\left(B_{1} v\right)^{\prime}(1)=0$ and $\left(B_{1} v\right)^{\prime \prime}(t)=-v(t)$. Integrating the right hand of the above by parts again gives (16). This completes the proof.

If both $h_{1}$ and $h_{2}$ are continuous and decreasing on $[0,1]$, then Chebyshev's inequality for $h_{1}$ and $h_{2}$ is given by

$$
\int_{0}^{1} h_{1}(t) h_{2}(t) \mathrm{d} t \geqslant \int_{0}^{1} h_{1}(t) \mathrm{d} t \int_{0}^{1} h_{2}(t) \mathrm{d} t .
$$

In addition, if both $h_{1}$ and $h_{2}$ are continuous, with one of them decreasing on $[0,1]$ and the other increasing on $[0,1]$, then Chebyshev's inequality for $h_{1}$ and $h_{2}$ becomes

$$
\int_{0}^{1} h_{1}(t) h_{2}(t) \mathrm{d} t \leqslant \int_{0}^{1} h_{1}(t) \mathrm{d} t \int_{0}^{1} h_{2}(t) \mathrm{d} t .
$$

Lemma 3. If $v \in P, v(1)=0$, and $v^{\prime}$ is decreasing on $[0,1]$, then

$$
\int_{0}^{1} v(t)(1-t) e^{1-t} d t \geqslant(e-2) \int_{0}^{1}-v^{\prime}(t)(1-t) e^{1-t} d t .
$$

Proof. Notice $v(1)=0$ and $v^{\prime} \leqslant 0$. Since $-v^{\prime}$ is increasing on $[0,1]$ and $(1-t) e^{1-t}$ is decreasing on $[0,1]$, it follows from Chebyshev's inequality that

$$
\begin{aligned}
\int_{0}^{1}-v^{\prime}(t)(1-t) e^{1-t} \mathrm{~d} t & \leqslant \int_{0}^{1}-v^{\prime}(t) \mathrm{d} t \int_{0}^{1}(1-t) e^{1-t} \mathrm{~d} t \\
& =v(0)
\end{aligned}
$$

Notice that $t e^{1-t}$ is increasing on $[0,1]$. Chebyshev's inequality again implies

$$
\int_{0}^{1}-v^{\prime}(t) t e^{1-t} \mathrm{~d} t \geqslant \int_{0}^{1}-v^{\prime}(t) \mathrm{d} t \int_{0}^{1} t e^{1-t} \mathrm{~d} t=(e-2) v(0) .
$$

Note that (15) is guaranteed by $v \in P, v(1)=0$. By virtue of (15), we have

$$
\int_{0}^{1} v(t)(1-t) e^{1-t} \mathrm{~d} t \geqslant(e-2) v(0) .
$$

Combining this with (22) yields (21). This complete the proof.

Lemma 4. If $v \in P, v(1)=0$, then

$$
v(0) \leqslant \int_{0}^{1}\left(v(t)-2 v^{\prime}(t)\right)(1-t) e^{1-t} \mathrm{~d} t .
$$

Proof. By (15), we have

$$
\begin{gathered}
\int_{0}^{1}\left(v(t)-2 v^{\prime}(t)\right)(1-t) e^{1-t} \mathrm{~d} s \\
=\int_{0}^{1}-v^{\prime}(t)(2-t) e^{1-t} \mathrm{~d} t \\
\geqslant \int_{0}^{1}-v^{\prime}(t) \mathrm{d} t=v(0)
\end{gathered}
$$

This completes the proof.
Lemma 5. If $v \in P \cap C^{2}[0,1]$ and $v^{\prime \prime} \leqslant 0$, then

$$
(e-2)\|v\|_{0} \leqslant \int_{0}^{1} v(t)(1-t) e^{1-t} \mathrm{~d} t \leqslant e\|v\|_{0} .
$$

Proof. It is easy to see that $\int_{0}^{1} v(t) t e^{t} \mathrm{~d} t \leqslant e\|v\|_{0}$. The condition $v^{\prime} \leqslant 0$ implies $\|v\|_{0}=v \leqslant(0)$. By the concavity of $v$, we have

$$
\begin{aligned}
\int_{0}^{1} v(t)(1-t) e^{1-t} \mathrm{~d} t & \geqslant \int_{0}^{1}(1-t) e^{1-t} v(t \cdot 1+(1-t) \cdot 0) \mathrm{d} t \\
& \geqslant v(0) \int_{0}^{1}(1-t)^{2} e^{1-t} \mathrm{~d} t=(e-2)\|v\|_{0} .
\end{aligned}
$$

This completes the proof.

Lemma 6. If $v \in P \cap C^{2}[0,1]$ and $v^{\prime \prime} \leqslant 0$, then we have

$$
\begin{aligned}
\frac{3-e}{6}\|v\|_{0} & \leqslant \int_{0}^{1}\left(\left(B_{1} v\right)(t)+2\left(B_{1} v\right)^{\prime}(t)\right)(1-t) e^{1-t} d t \\
& \leqslant 3 e\|v\|_{0} .
\end{aligned}
$$

Proof. By the concavity of $B_{1} v$ and $v$, we have

$$
\begin{aligned}
& \int_{0}^{1}\left(\left(B_{1} v\right)(t)+2\left(B_{1} v\right)^{\prime}(t)\right)(1-t) e^{1-t} \mathrm{~d} t \\
& \geqslant \int_{0}^{1}\left(B_{1} v\right)(t)(1-t) e^{1-t} \mathrm{~d} t \\
& =\int_{0}^{1}\left(B_{1} v\right)(t \cdot 1+(1-t) \cdot 0)(1-t) e^{1-t} \mathrm{~d} t \\
& \geqslant\left(B_{1} v\right)(1) \int_{0}^{1} t(1-t) e^{1-t} \mathrm{~d} t \\
& =(3-e) \int_{0}^{1} s v(s) \mathrm{d} s \\
& \geqslant(3-e) \int_{0}^{1} s(1-s) v(0) \mathrm{d} s \\
& =\frac{3-e}{6}\|v\|_{0},
\end{aligned}
$$

$$
\begin{aligned}
& \int_{0}^{1}\left(\left(B_{1} v\right)(t)+2\left(B_{1} v\right)^{\prime}(t)\right)(1-t) e^{1-t} \mathrm{~d} t \\
& \quad \leqslant e \int_{0}^{1}\left(B_{1} v\right)(t)+2\left(B_{1} v\right)^{\prime}(t) \mathrm{d} t \\
& \quad=e \int_{0}^{1}\left(\int_{0}^{1} k_{1}(t, s) v(s) \mathrm{d} s+2 \int_{0}^{1} v(s) \mathrm{d} s\right) \mathrm{d} t \\
& \quad \leqslant 3 e\|v\|_{0} .
\end{aligned}
$$

This completes the proof.

Lemma 7 (see [19]). Let $E$ be a real Banach space and $P$ a cone in $E$. Suppose that $\Omega \subset E$ is a bounded open set and that 
$T: \bar{\Omega} \cap P \rightarrow P$ is a completely continuous operator. If there exists $\omega_{0} \in P \backslash\{0\}$ such that

$$
\omega-T \omega \neq \lambda \omega_{0}, \quad \forall \lambda \geqslant 0, \omega \in \partial \Omega \cap P,
$$

then $i(T, \Omega \cap P, P)=0$, where $i$ indicates the fixed point index on $P$.

Lemma 8 (see [19]). Let $E$ be a real Banach space and $P$ a cone in $E$. Suppose that $\Omega \subset E$ is a bounded open set with $0 \in \Omega$ and that $T: \bar{\Omega} \cap P \rightarrow P$ is a completely continuous operator. If

$$
\omega-\lambda T \omega \neq 0, \quad \forall \lambda \in[0,1], \omega \in \partial \Omega \cap P,
$$

then $i(T, \Omega \cap P, P)=1$.

\section{Existence and Multiplicity of Positive Solutions for (1)}

For simplicity, we denote by $x:=\left(x_{1}, x_{2}, x_{3}, x_{4}\right) \in \mathbb{R}_{+}^{4}$ and $I_{\rho}:=[0, \rho]$ for $\rho>0$ in this section. Now we list our hypotheses.

(H1) $f \in C\left([0,1] \times \mathbb{R}_{+}^{4}, \mathbb{R}_{+}\right)$.

(H2) There exist $a_{1} \geqslant 0, b_{1} \geqslant 0, c>0$, such that $d_{1}:=$ $a_{1}(3-e(e-2)) / 6 e^{2}+b_{1}>1$, and

$$
f(t, x) \geqslant a_{1}\left(x_{1}+2 x_{2}\right)+b_{1}\left(x_{3}+2 x_{4}\right)-c
$$

holds for all $(t, x) \in[0,1] \times \mathbb{R}_{+}^{4}$.

(H3) There exists a function $\Phi \in C\left(\mathbb{R}_{+}, \mathbb{R}_{+}\right)$such that

$$
f(t, x) \leqslant \Phi\left(x_{4}\right)
$$

for all $x \in[0,1] \times I_{m}^{3} \times \mathbb{R}_{+}$and

$$
\int_{0}^{\infty} \frac{\xi \mathrm{d} \xi}{\Phi(\xi)+2 m e}>m .
$$

where $m:=c /\left(d_{1}-1\right)>0$ and $c, d_{1}$ are given as in (H2).

(H4) There exist $a_{2} \geqslant 0, b_{2} \geqslant 0, r>0$ such that $d_{2}:=$ $3 a_{2} e /(e-2)+b_{2}<1$, and

$$
f(t, x) \leqslant a_{2}\left(x_{1}+2 x_{2}\right)+b_{2}\left(x_{3}+2 x_{4}\right)
$$

holds for all $(t, x) \in[0,1] \times I_{r}^{3}$.

(H5) There exist $a_{3} \geqslant 0, b_{3} \geqslant 0, c>0$ such that $d_{3}:=$ $3 a_{3} e /(e-2)+b_{2}<1$, and

$$
f(t, x) \leqslant a_{3}\left(x_{1}+2 x_{2}\right)+b_{3}\left(x_{3}+2 x_{4}\right)+c
$$

holds for all $(t, x) \in[0,1] \times \mathbb{R}_{+}^{4}$.

(H6) There are nonnegative constants $a_{4} \geqslant 0, b_{4} \geqslant 0, r>0$, such that $d_{4}:=a_{4}(3-e)(e-2) / 6 e^{2}+b_{4}>1$, and

$$
f(t, x) \geqslant a_{4}\left(x_{1}+2 x_{2}\right)+b_{4}\left(x_{3}+2 x_{4}\right)
$$

holds for all $(t, x) \in[0,1] \times I_{r}^{4}$.
(H7) There is a constant $\omega>0$ such that $f(t, x)$ is increasing on $I_{\omega}^{4}$ in $x$, and

$$
\int_{0}^{1} f(s, \omega, \omega, \omega, \omega) \mathrm{d} s<\omega .
$$

Remark 9. $f(t, x)$ is said to be increasing in $x$ if $f(t, x) \leqslant$ $f(t, y)$ holds for every pair $x, y \in \mathbb{R}_{+}^{4}$ with $x \leqslant y$ and for all $t \in[0,1]$, where the partial ordering in $\leqslant \mathbb{R}^{4}$ is understood componentwise. sequel.

We denote $\Omega_{\rho}:=\{v \in E:\|v\|<\rho\}$ for $\rho>0$ in the

Theorem 10. If (H1)-(H4) hold, then (1) has at least one positive solution.

Proof. Let

$$
\mathscr{M}_{1}:=\{v \in P: v=A v+\mu \varphi, \mu \geqslant 0\}
$$

where $\varphi(t):=(1-t) e^{t-1} \in P$. We are now going to prove that $\mathscr{M}_{1}$ is bounded. Indeed, if $v \in \mathscr{M}_{1}$, then there exist $\mu \geqslant 0$ such that $v=A v+\mu \varphi$, which can be written in the form

$$
\begin{aligned}
& v(t) \\
& =\int_{0}^{1} k_{2}(t, s) f\left(s,\left(B_{1} v\right)(s),\left(B_{1} v\right)^{\prime}(s), v(s),-v^{\prime}(s)\right) \mathrm{d} s \\
& \quad+\mu \varphi(t),
\end{aligned}
$$

which is equivalent to

$$
\begin{aligned}
-v^{\prime \prime}(t)= & f\left((t),\left(B_{1} v\right)(t),\left(B_{1} v\right)^{\prime}(t), v(t),-v^{\prime}(t)\right) \\
& +\mu(1+t) e^{t-1} .
\end{aligned}
$$

By $(\mathrm{H} 2)$, we have

$$
\begin{aligned}
-v^{\prime \prime}(t) \geqslant & a_{1}\left(\left(B_{1} v\right)(t)+2\left(B_{1} v\right)^{\prime}(t)\right) \\
& +b_{1}\left(v(t)-2 v^{\prime}(t)\right)-c .
\end{aligned}
$$


Note (27) and (29). Multiply the above by $(1-t) e^{1-t}$, integrate over $[0,1]$, and use Lemmas 3,5 , and 6 to obtain

$$
\begin{aligned}
& \int_{0}^{1}\left(v(t)-2 v^{\prime}(t)\right)(1-t) e^{1-t} \mathrm{~d} t \\
& \geqslant a_{1} \int_{0}^{1}\left(\left(B_{1} v\right)(t)+2\left(B_{1} v\right)^{\prime}(t)\right)(1-t) e^{1-t} \mathrm{~d} t \\
&+b_{1} \int_{0}^{1}\left(v(t) 2 v^{\prime}(t)\right)(1-t) e^{1-t} \mathrm{~d} t-c \\
& \geqslant \frac{a_{1}(3-e)}{6}\|v\|_{0} \\
&+b_{1} \int_{0}^{1}\left(v(t)-2 v^{\prime}(t)\right)(1-t) e^{1-t} \mathrm{~d} t-c \\
& \geqslant \frac{a_{1}(3-e)}{6 e} \int_{0}^{1} v(t)(1-t) e^{1-t} \mathrm{~d} t \\
&+b_{1} \int_{0}^{1}\left(v(t)-2 v^{\prime}(t)\right)(1-t) e^{1-t} \mathrm{~d} t-c \\
& \geqslant d_{1} \int_{0}^{1}\left(v(t)-2 v^{\prime}(t)\right)(1-t) e^{1-t} \mathrm{~d} t-c
\end{aligned}
$$

so that

$$
\int_{0}^{1}\left(v(t)-2 v^{\prime}(t)\right)(1-t) e^{1-t} \mathrm{~d} t \leqslant m, \quad \forall v \in \mathscr{M}_{1} .
$$

Now Lemma 4 implies

$$
\|v\|_{0}=v(0) \leqslant m, \quad \forall v \in \mathscr{M}_{1},
$$

where $m$ is given in (H3). Furthermore, this estimate leads to

$$
\begin{aligned}
& \left\|\left(B_{1} v\right)(t)\right\|_{0}=\left(B_{1} v\right)(1)=\int_{0}^{1} s v(s) \mathrm{d} s \leqslant m, \\
& \left\|\left(B_{1} v\right)^{\prime}(t)\right\|_{0}=\left(B_{1} v\right)^{\prime}(0)=\int_{0}^{1} v(s) \mathrm{d} s \leqslant m
\end{aligned}
$$

for all $v \in \mathscr{M}_{1}$. Let

$$
\begin{array}{r}
\Lambda:=\{\mu \geqslant 0: \text { there exist some } v \in P \\
\text { such that } v=A v+\mu \varphi\} .
\end{array}
$$

Now (46) implies that $\mu \leqslant m e$ for all $\mu \in \Lambda$. By (H3), there is a function $\Phi \in C\left(\mathbb{R}_{+}, \mathbb{R}_{+}\right)$such that

$$
\begin{aligned}
& f\left(t,\left(B_{1} v\right)(t),\left(B_{1} v\right)^{\prime}(t), v(t),-v^{\prime}(t)\right) \\
& \leqslant \Phi\left(-v^{\prime}(t)\right), \quad \forall v \in \mathscr{M}_{1} .
\end{aligned}
$$

\section{Thus}

$$
\begin{aligned}
-v^{\prime \prime}(t)= & f\left(t,\left(B_{1} v\right)(t),\left(B_{1} v\right)^{\prime}(t), v(t),-v^{\prime}(t)\right) \\
& +\mu(1+t) e^{t-1} \\
\leqslant & \Phi\left(-v^{\prime}(t)\right)+\mu(1+t) e^{t-1} \\
\leqslant & \Phi\left(-v^{\prime}(t)\right)+2 m e, \quad \forall v \in \mathscr{M}_{1}, \mu \in \Lambda, \\
& \int_{0}^{-v^{\prime}(1)} \frac{\xi \mathrm{d} \xi}{\Phi(\xi)+2 m e} \\
& \leqslant \int_{0}^{1}-v^{\prime}(t) \mathrm{d} t=v(0) \leqslant m, \quad \forall v \in \mathscr{M}_{1} .
\end{aligned}
$$

Combining the last inequality with (35), we see that there exists $M>0$ such that $-v(1) \leqslant M$ for all $v \in \mathscr{M}_{1}$. This means that $\mathscr{M}_{1}$ is bounded. Taking $R>\sup \left\{\|v\|: v \in \mathscr{M}_{1}\right\}$, we have

$$
v \neq A v+\mu \varphi, \quad \forall v \in \Omega_{R} \cap P, \mu \geq 0 .
$$

Now Lemma 7 yields

$$
i\left(A, \Omega_{R} \cap P, P\right)=0 .
$$

Let

$$
\mathscr{M}_{2}:=\left\{v \in \bar{\Omega}_{r} \cap P: v=\lambda A v, \lambda \in[0,1]\right\} .
$$

Now we want to prove that $\mathscr{M}_{2}=\{0\}$. In fact, if $v \in \mathscr{M}_{2}$, then $v \in \bar{\Omega}_{r} \cap P$ and there is $\lambda \in[0,1]$ such that $v=\lambda A v$, that is,

$$
\begin{aligned}
v(t)= & \lambda \int_{0}^{1} k_{2}(t, s) \\
& \times f\left(s,\left(B_{1} v\right)(s),\left(B_{1} v\right)^{\prime}(s), v(s),-v^{\prime}(s)\right) \mathrm{d} s,
\end{aligned}
$$

which can be written in the form

$$
-v^{\prime \prime}(t)=\lambda f\left(t,\left(B_{1} v\right)(t),\left(B_{1} v\right)^{\prime}(t), v(t),-v^{\prime}(t)\right) .
$$

By (H4), we have

$$
-v^{\prime \prime}(t) \leqslant a_{2}\left(\left(B_{1} v\right)(t)+2\left(B_{1} v\right)^{\prime}(t)\right)+b_{2}\left(v(t)-2 v^{\prime}(t)\right) .
$$


Note (27) and (29). Multiply (56) by $(1-t) e^{1-t}$, integrate over $[0,1]$, and use Lemmas 5 and 6 to obtain

$$
\begin{aligned}
& \int_{0}^{1}\left(v(t)-2 v^{\prime}(t)\right)(1-t) e^{1-t} \mathrm{~d} t \\
& \leqslant a_{2} \int_{0}^{1}\left(\left(B_{1} v\right)(t)+2\left(B_{1} v\right)^{\prime}(t)\right)(1-t) e^{1-t} \mathrm{~d} t \\
&+b_{2} \int_{0}^{1}\left(v(t)-2 v^{\prime}(t)\right)(1-t) e^{1-t} \mathrm{~d} t \\
& \leqslant 3 e a_{2}\|v\|_{0}+b_{2} \int_{0}^{1}\left(v(t)-2 v^{\prime}(t)\right)(1-t) e^{1-t} \mathrm{~d} t \\
& \leqslant \frac{3 e a_{2}}{e-2} \int_{0}^{1} v(t)(1-t) e^{1-t} \mathrm{~d} t \\
&+b_{2} \int_{0}^{1}\left(v(t)-2 v^{\prime}(t)\right)(1-t) e^{1-t} \mathrm{~d} t \\
& \leqslant d_{2} \int_{0}^{1}\left(v(t)-2 v^{\prime}(t)\right)(1-t) e^{1-t} \mathrm{~d} t,
\end{aligned}
$$

so that $\int_{0}^{1}\left(v(t)-2 v^{\prime}(t)\right)(1-t) e^{1-t}=0$, whence $v(t) \equiv 0$ and $\mathscr{M}_{2}=\{0\}$, as required. A consequence of this is

$$
v \neq \lambda A v, \quad \forall v \in \partial \Omega_{r} \cap P, \lambda \in[0,1] .
$$

Now Lemma 8 yields

$$
i\left(A, \Omega_{r} \cap P, P\right)=1 \text {. }
$$

This together with (52) implies

$$
i\left(A,\left(\Omega_{R} \backslash \bar{\Omega}_{r}\right) \cap P, P\right)=0-1=-1 .
$$

Therefore $A$ has at least one fixed point on $\left(\Omega_{R} \backslash \bar{\Omega}_{r}\right) \cap P$ and thus (1) has at least one positive solution. This completes the proof.

Theorem 11. If(H1), (H5), and (H6) hold, then (1) has at least one positive solution.

Proof. Let

$$
\mathscr{M}_{3}:=\{v \in P: v=\lambda A v, \lambda \in[0,1]\}
$$

We now assert that $\mathscr{M}_{3}$ is bounded. Indeed, if $v \in \mathscr{M}_{3}$, then there is $\lambda \in[0,1]$ such that $v=\lambda A v$, which is equivalent to

$$
-v^{\prime \prime}(t)=\lambda f\left(t,\left(B_{1} v\right)(t),\left(B_{1}\right)^{\prime}(t), v(t),-v^{\prime}(t)\right) \text {. }
$$

Now (H5) implies

$$
\begin{aligned}
-v^{\prime \prime}(t) \leqslant & a_{3}\left(\left(B_{1} v\right)(t)+2\left(B_{1} v\right)^{\prime}(t)\right) \\
& +b_{3}\left(v(t)-2 v^{\prime}(t)\right)+c .
\end{aligned}
$$

Note (27) and (29). Multiply the above by $(1-t) e^{1-t}$, integrate over $[0,1]$, and use Lemmas 5 and 6 to obtain

$$
\begin{aligned}
\int_{0}^{1}\left(v(t)-2 v^{\prime}(t)\right)(1-t) e^{1-t} \mathrm{~d} t \\
\leqslant a_{3} \int_{0}^{1}\left(\left(B_{1} v\right)(t)+2\left(B_{1} v\right)^{\prime}(t)\right)(1-t) e^{1-t} \mathrm{~d} t \\
\quad+b_{3} \int_{0}^{1}\left(v(t)-2 v^{\prime}(t)\right)(1-t) e^{1-t} \mathrm{~d} t+c \\
\leqslant 3 e a_{3}\|v\|_{0}+b_{3} \int_{0}^{1}\left(v(t)-2 v^{\prime}(t)\right)(1-t) e^{1-t} \mathrm{~d} t+c \\
\leqslant d_{3} \int_{0}^{1}\left(v(t)-2 v^{\prime}(t)\right)(1-t) e^{1-t} \mathrm{~d} t+c,
\end{aligned}
$$

so that $\int_{0}^{1}\left(v(t)-2 v^{\prime}(t)\right)(1-t) e^{1-t} \mathrm{~d} t \leqslant c_{1}$ for all $v \in \mathscr{M}_{3}$, where $c_{1}:=c /\left(1-d_{3}\right)>0$. By Lemma 4 , we obtain

$$
\begin{aligned}
\|v\|_{0} & =v(0) \leqslant \int_{0}^{1}\left(v(t)-2 v^{\prime}(t)\right)(1-t) e^{1-t} \mathrm{~d} t \\
& \leqslant c_{1}, \quad \forall v \in \mathscr{M}_{3} .
\end{aligned}
$$

Furthermore, this estimate leads to

$$
\begin{gathered}
\left\|B_{1} v\right\|_{0}=\left(B_{1} v\right)(1)=\int_{0}^{1} s v(s) \mathrm{d} s \leqslant c_{1}, \\
\left\|\left(B_{1} v\right)^{\prime}\right\|_{0}=\left(B_{1} v\right)^{\prime}(0)=\int_{0}^{1} v(s) \mathrm{d} s \leqslant c_{1}
\end{gathered}
$$

for all $v \in \mathscr{M}_{3}$. By (63), we have

$$
-v^{\prime \prime}(t) \leqslant\left(3 a_{3}+b_{3}\right) c_{1}-2 b_{3} v^{\prime}(t)+c, \quad \forall v \in \mathscr{M}_{3} .
$$

Note $v(1)=v^{\prime}(0)=0$ and $v^{\prime \prime} \leqslant 0$. Integrating the above over $[0,1]$, we obtain

$$
\begin{aligned}
\left\|v^{\prime}\right\|_{0} & =-v^{\prime}(1) \leqslant\left(3 a_{3}+b_{3}\right) c_{1}+2 b_{3} v(0)+c \\
& \leqslant 3\left(a_{3}+b_{3}\right) c_{1}+c .
\end{aligned}
$$

This proves the boundedness of $\mathscr{M}_{3}$. Taking $R>\sup \{\|v\|$ : $\left.v \in \mathscr{M}_{3}\right\}$, we have

$$
v \neq \lambda A v, \quad \forall v \in \partial \Omega_{R} \cap P, \lambda \in[0,1] .
$$

Now Lemma 8 yields

$$
i\left(A, \Omega_{R} \cap P, P\right)=1 .
$$

On the other hand, let

$$
\mathscr{M}_{4}:=\left\{v \in \bar{\Omega}_{r} \cap P: v=A v+\lambda \varphi, \lambda \geqslant 0\right\},
$$

where $\varphi \in P \backslash\{0\}$ is given by $\varphi(t):=(1-t) e^{t-1}$. Now we want to prove that $\mathscr{M}_{4} \subset\{0\}$. Indeed, if $v \in \mathscr{M}_{4}$, then there exists $\lambda \geqslant 0$ such that $v=A v+\lambda \varphi$, which can be written as

$$
\begin{aligned}
-v^{\prime \prime}(t)= & f\left(t,\left(B_{1} v\right)(t),\left(B_{1} v\right)^{\prime}(t), v(t),-v^{\prime}(t)\right) \\
& +\lambda(t+1) e^{t-1} .
\end{aligned}
$$


By (H6), we have

$$
-v^{\prime \prime}(t) a_{4} \geqslant\left(\left(B_{1} v\right)(t)+2\left(B_{1} v\right)^{\prime}(t)\right)+b_{4}\left(v(t)-2 v^{\prime}(t)\right) .
$$

Note (27) and (29). Multiply the above by $(1-t) e^{1-t}$, integrate over $[0,1]$, and use Lemmas 3, 5, and 6 to obtain

$$
\begin{aligned}
& \int_{0}^{1}\left(v(t)-2 v^{\prime}(t)\right)(1-t) e^{1-t} \mathrm{~d} t \\
& \geqslant a_{4} \int_{0}^{1}\left(\left(B_{1} v\right)(t)+2\left(B_{1} v\right)^{\prime}(t)\right)(1-t) e^{1-t} \mathrm{~d} t \\
&+b_{4} \int_{0}^{1}\left(v(t)-2 v^{\prime}(t)\right)(1-t) e^{1-t} \mathrm{~d} t \\
& \geqslant \frac{a_{4}(3-e)}{6 e}\|v\|_{0}+b_{4} \int_{0}^{1}\left(v(t)-2 v^{\prime}(t)\right)(1-t) e^{1-t} \mathrm{~d} t \\
& \geqslant \frac{a_{4}(3-e)}{6 e} \int_{0}^{1} v(t)(1-t) e^{1-t} \mathrm{~d} t \\
&+b_{4} \int_{0}^{1}\left(v(t)-2 v^{\prime}(t)\right)(1-t) e^{1-t} \mathrm{~d} t \\
& \geqslant d_{4} \int_{0}^{1}\left(v(t)-2 v^{\prime}(t)\right)(1-t) e^{1-t} \mathrm{~d} t
\end{aligned}
$$

so that $\int_{0}^{1}\left(v(t)-2 v^{\prime}(t)\right)(1-t) e^{1-t} \mathrm{~d} t=0$, whence $v(t) \equiv 0$. This proves $\mathscr{M}_{4} \subset\{0\}$, as required. As a result of this, we obtain

$$
v \neq A v+\lambda \varphi, \quad \forall v \in \partial \Omega_{r} \cap P, \lambda \geqslant 0 .
$$

Lemma 7 implies

$$
i\left(A, \Omega_{r} \cap P, P\right)=0 .
$$

Now (70) and (76) combined lead to

$$
i\left(A,\left(\Omega_{R} \backslash \bar{\Omega}_{r}\right) \cap P, P\right)=1-0=1 .
$$

Consequently, $A$ has at least one fixed point on $\left(\Omega_{R} \backslash \bar{\Omega}_{r}\right) \cap P$. Thus (1) has at least one positive solution. This completes the proof.

Theorem 12. If (H1), (H2), (H3), (H6), and (H7) hold, then (1) has at least two positive solutions.

Proof. If $v \in P$ and $\|v\| \leqslant \omega$, then we have

$$
\begin{aligned}
\left\|B_{1} v\right\|_{0} & =\left(B_{1} v\right)(1)=\int_{0}^{1} s v(s) \mathrm{d} s \leqslant \omega,\left\|\left(B_{1} v\right)^{\prime}\right\|_{0}=\left(B_{1} v\right)(0) \\
& =\int_{0}^{1} v(s) \mathrm{d} s \leqslant \omega .
\end{aligned}
$$

By (H7), we have

$$
f(t, x) \leqslant f(t, \omega, \omega, \omega, \omega), \quad \forall t \in[0,1], x \in I_{\omega}^{4} .
$$

Consequently, for all $v \in \partial \Omega_{\omega} \cap P$, we have

$$
\begin{aligned}
&\|A v\|_{0}=(A v)(0) \\
&=\int_{0}^{1}(1-s) f\left(s,\left(B_{1} v\right)(s),\left(B_{1} v\right)^{\prime}(s) v(s),-v^{\prime}(s)\right) \mathrm{d} s \\
& \leqslant \int_{0}^{1} f(s, \omega, \omega, \omega, \omega) \mathrm{d} s<\omega=\|v\|, \\
&\left\|(A v)^{\prime}\right\| \\
& \quad=-(A v)^{\prime}(1) \\
& \quad=\int_{0}^{1} f\left(s,\left(B_{1} v\right)(s),\left(B_{1} v\right)^{\prime}(s), v(s),-v^{\prime}(s)\right) \mathrm{d} s \\
& \quad \leqslant \int_{0}^{1} f(s, \omega, \omega, \omega, \omega) \mathrm{d} s<\omega=\|v\| .
\end{aligned}
$$

The preceding inequalities imply

$$
\|A v\|<\omega=\|v\|, \quad \forall v \in \partial \Omega_{\omega} \cap P
$$

and thus

$$
v \neq \lambda A v, \quad \forall v \in \partial \Omega_{\omega} \cap P, 0 \leqslant \lambda \leqslant 1 .
$$

Now Lemma 5 yields

$$
i\left(A, \Omega_{\omega} \cap P, P\right)=1 .
$$

By (H2), (H3), and (H6), we know that (52) and (76) hold (see the proofs of Theorems 10 and 11). Note that we can choose $R>\omega>r$ in (52) and (76). Combining (52), (76), and (84), we obtain

$$
\begin{gathered}
i\left(A,\left(\Omega_{R} \backslash \bar{\Omega}_{\omega}\right) \cap P, P\right)=0-1=-1, \\
i\left(A,\left(\Omega_{\omega} \backslash \bar{\Omega}_{r}\right) \cap P, P\right)=1-0=1 .
\end{gathered}
$$

Therefore, $A$ has at least two fixed points, with one on $\left(\Omega_{R}\right)$ $\left.\bar{\Omega}_{\omega}\right) \cap P$ and the other on $\left(\Omega_{\omega} \backslash \bar{\Omega}_{r}\right) \cap P$. Hence (1) has at least two positive solutions. This completes the proof.

\section{References}

[1] T. Jankowski and R. Jankowski, "Multiple solutions of boundary-value problems for fourth-order differential equations with deviating arguments," Journal of Optimization Theory and Applications, vol. 146, no. 1, pp. 105-115, 2010.

[2] C. Zhai, R. Song, and Q. Han, "The existence and the uniqueness of symmetric positive solutions for a fourth-order boundary value problem," Computers \& Mathematics with Applications, vol. 62, pp. 2639-2647, 2011.

[3] B. Yang, "Positive solutions for the beam equation under certain boundary conditions," Electronic Journal of Differential Equations, vol. 78, pp. 1-8, 2005. 
[4] Q. Yao, "Positive solutions for eigenvalue problems of fourthorder elastic beam equations," Applied Mathematics Letters, vol. 17, no. 2, pp. 237-243, 2004.

[5] Z. Wei and C. Pang, "Multiple sign-changing solutions for fourth order m-point boundary value problems," Nonlinear Analysis, Theory, Methods and Applications, vol. 66, no. 4, pp. 839-855, 2007.

[6] L. Liu, P. Kang, Y. Wu, and B. Wiwatanapataphee, "Positive solutions of singular boundary value problems for systems of nonlinear fourth order differential equations," Nonlinear Analysis, Theory, Methods and Applications, vol. 68, no. 3, pp. 485-498, 2008.

[7] Z. Bai, "The upper and lower solution method for some fourthorder boundary value problems," Nonlinear Analysis, Theory, Methods and Applications, vol. 67, no. 6, pp. 1704-1709, 2007.

[8] Q. Zhang, S. Chen, and J. Lü, "Upper and lower solution method for fourth-order four-point boundary value problems," Journal of Computational and Applied Mathematics, vol. 196, no. 2, pp. 387-393, 2006.

[9] D. X. Ma and X. Z. Yang, "Upper and lower solution method for fourth-order four-point boundary value problems," Journal of Computational and Applied Mathematics, vol. 223, no. 2, pp. 543-551, 2009.

[10] J. R. Graef and L. Kong, "A necessary and sufficient condition for existence of positive solutions of nonlinear boundary value problems," Nonlinear Analysis, Theory, Methods and Applications, vol. 66, no. 11, pp. 2389-2412, 2007.

[11] R. P. Agarwal and Y. M. Chow, "Iterative methods for a fourth order boundary value problem," Journal of Computational and Applied Mathematics, vol. 10, no. 2, pp. 203-217, 1984.

[12] Q. Yao, "Monotone iterative technique and positive solutions of Lidstone boundary value problems," Applied Mathematics and Computation, vol. 138, no. 1, pp. 1-9, 2003.

[13] E. Alves, T. F. Ma, and M. L. Pelicer, "Monotone positive solutions for a fourth order equation with nonlinear boundary conditions," Nonlinear Analysis, Theory, Methods and Applications, vol. 71, no. 9, pp. 3834-3841, 2009.

[14] M. Pei and S. K. Chang, "Monotone iterative technique and symmetric positive solutions for a fourth-order boundary value problem," Mathematical and Computer Modelling, vol. 51, no. 910, pp. 1260-1267, 2010.

[15] G. Han and F. Li, "Multiple solutions of some fourth-order boundary value problems," Nonlinear Analysis, Theory, Methods and Applications, vol. 66, no. 11, pp. 2591-2603, 2007.

[16] P. Amster and P. P. Cárdenas Alzate, "A shooting method for a nonlinear beam equation," Nonlinear Analysis, Theory, Methods and Applications, vol. 68, no. 7, pp. 2072-2078, 2008.

[17] C. Pang, W. Dong, and Z. Wei, "Multiple solutions for fourthorder boundary value problem," Journal of Mathematical Analysis and Applications, vol. 314, no. 2, pp. 464-476, 2006.

[18] Z. Yang and J. Sun, "Positive solutions of a fourth-order boundary value problem involving derivatives of all orders," Communications on Pure and Applied Analysis, vol. 11, no. 5, pp. 1615-1628, 2012.

[19] D. Guo and V. Lakshmikantham, Problems in Abstract Cones, Academic Press, Boston, Mass, USA, 1988. 


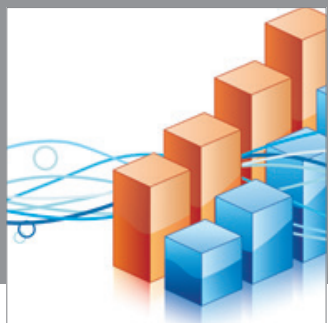

Advances in

Operations Research

mansans

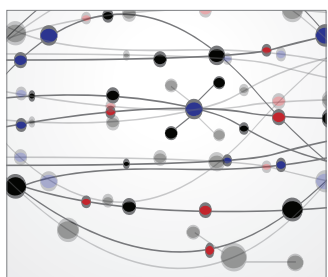

The Scientific World Journal
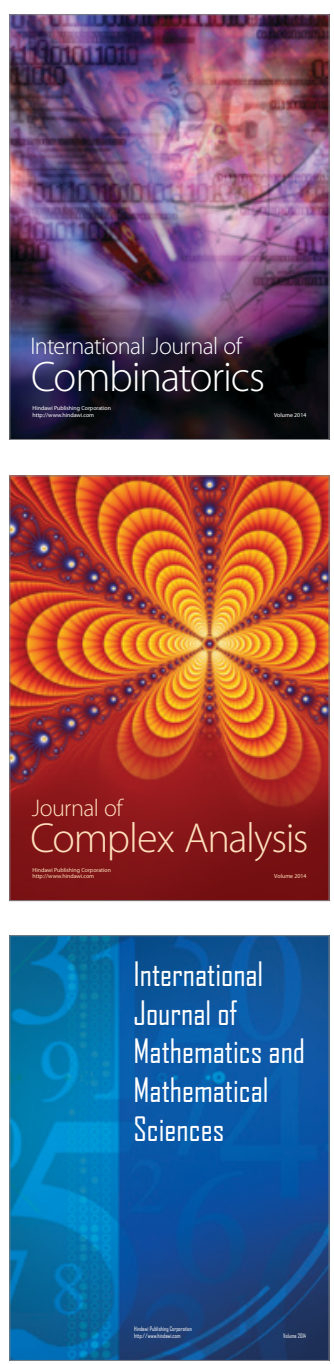
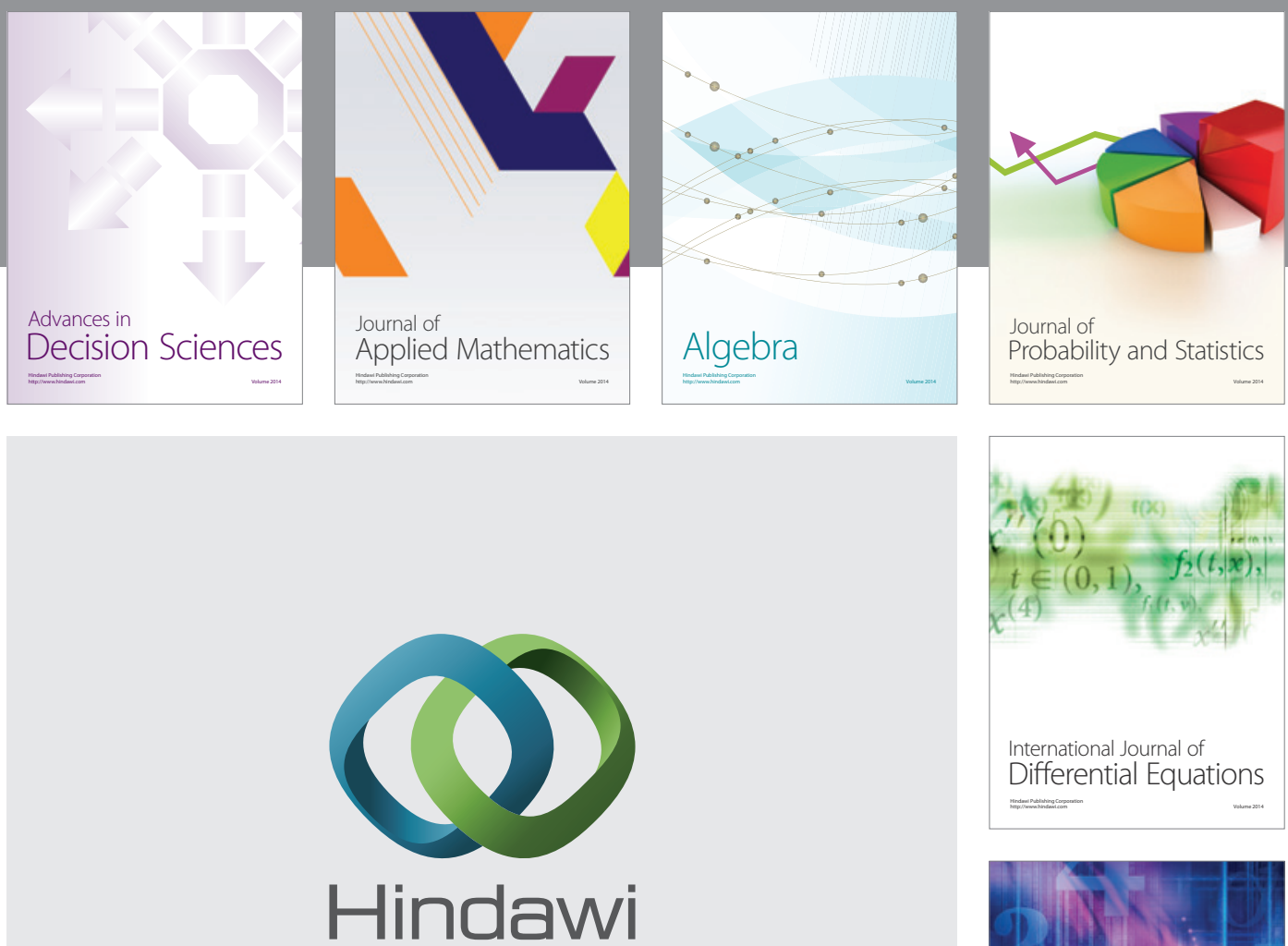

Submit your manuscripts at http://www.hindawi.com
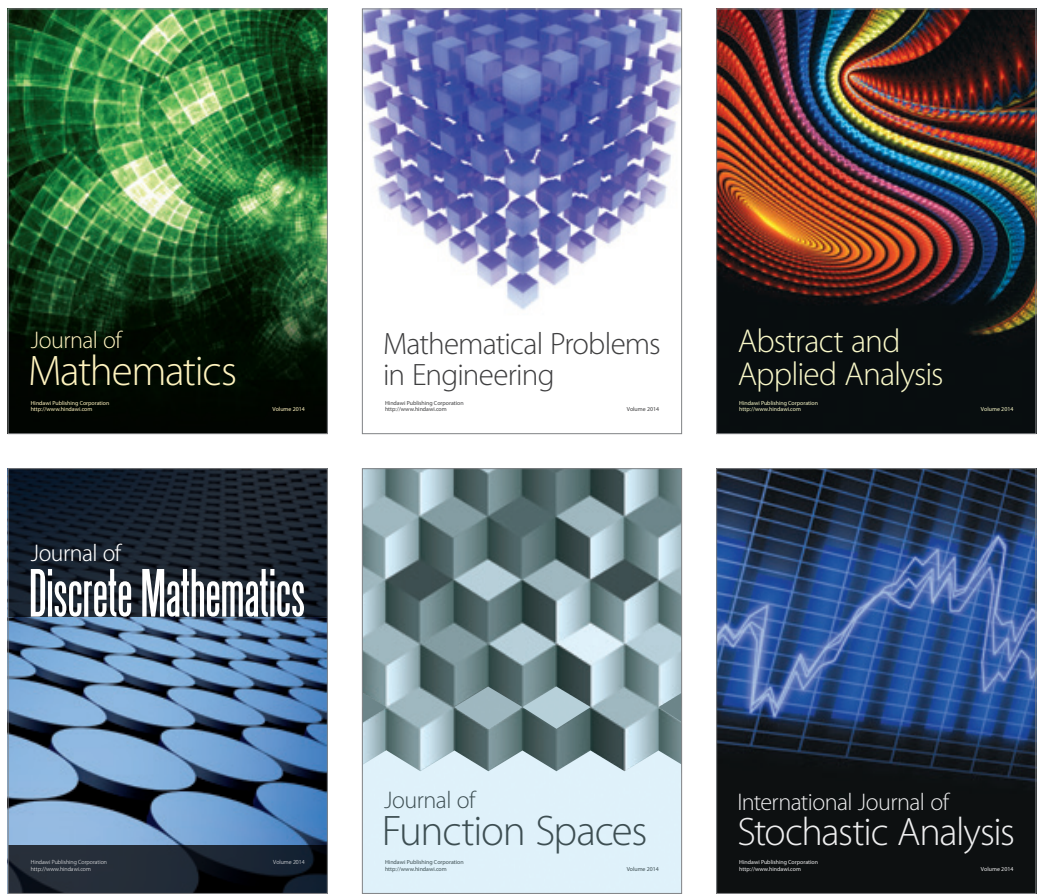

Journal of

Function Spaces

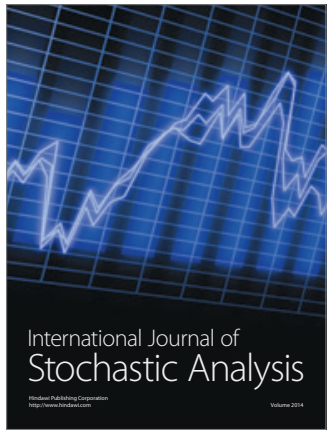

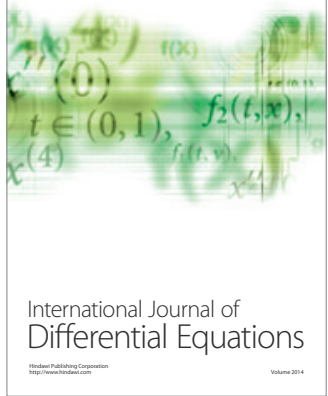
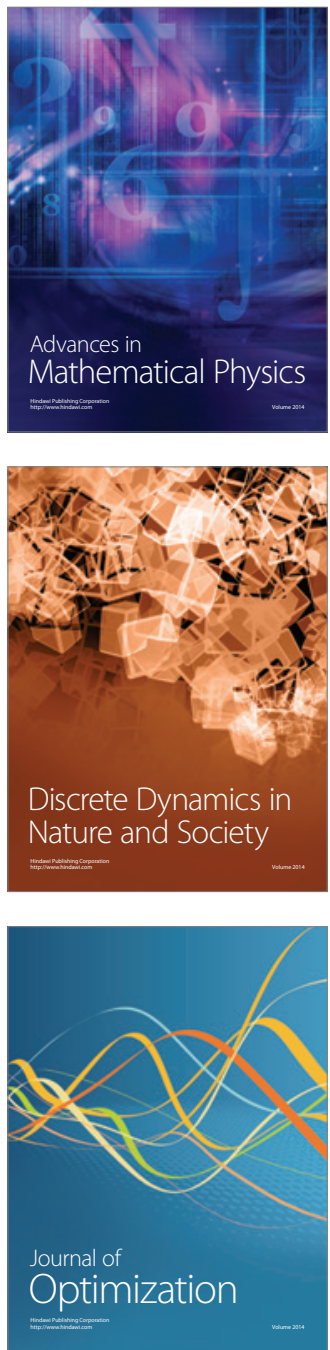